\title{
Heart Rate Variability Analysis of Normal and Growth Restricted Children
}

\section{Wajid Aziz $^{1,3}$, Taher Biala ${ }^{1}$, Federico Cardona Rocha ${ }^{1}$, Michael Wailoo ${ }^{2}$, Fernando S. Schlindwein ${ }^{1}$}

\footnotetext{
${ }^{1}$ University of Leicester, Department of Engineering

${ }^{2}$ Leicester Royal Infirmary and University of Leicester, Child Health Department

${ }^{3}$ Department of Computer Sciences and Information Technology AJK University Muzaffarabad (AK), Pakistan
}

\begin{abstract}
Intrauterine growth restriction (IUGR) has been associated with an increased risk of cardiovascular disease in later life. The regularity mechanism of cardiovascular system is under the control of autonomic nervous system (ANS). The non-optimal fetal growth may alter the development of the ANS and this appears to persist in later life. The aim of the present work is to analyse the synergic activity of the ANS in normal and growth restricted children. For that purpose, heart rate variability analysis from 24 hour ECG recordings of 70 children between 9 and 10 years old, normal and IUGR was performed using linear and non-linear time series analysis techniques. The HRV parameters showed no significant difference between normal and IUGR children. Low birth weight and its association with development of the cardiovascular system and its control have been extensively studied. In order to investigate the effect of low birth weight on HRV parameters, the IUGR children were further divided into two groups: IUGR-1 (birth weight $<2.50 \mathrm{~kg}$ ) and IUGR-2 (birth weight $\geq 2.50 \mathrm{~kg}$ ). The results demonstrated that most of the HRV measures showed significant differences between normal $V s$. IUGR-1 as well as IUGR-1 $V s$. IUGR-2 groups. The effect of gender on HRV measures were also examined and we noticed that girls had lower HRV than boys.
\end{abstract}

Keywords: Autonomic nervous system, Coronary heart disease, HRV analysis, IUGR, Low birth weight.

\section{Introduction}

Fetal growth restriction, also known as intrauterine growth restriction (IUGR), complicates $3 \%$ to $10 \%$ of all pregnancies. Low birth weight (LBW), premature birth and small for gestational age (SGA) are the markers to monitor intrauterine growth restriction (IUGR) and adverse prenatal outcomes [8]. A low birth weight infant is one born weighing < $2.5 \mathrm{~kg}[12,30]$. An infant born less than 37 weeks from first day of menstrual period 
regardless of birth weight is preterm, whereas the growth retarded infants are characterized by their biometric dimension $<10^{\text {th }}$ percentile for a given gestational age[12]. IUGR children are reported to suffer from increased incidence of intrauterine fetal death (IUFD), sudden death in infancy, cognitive dysfunction during childhood, altered autonomic nervous system (ANS), increased incidence of obesity and cardiovascular morbidity during adulthood. Geva et al. (2006) evaluated the effect of late onset of intrauterine growth restriction on neuropsychological profile and academic achievements at 9 years of age [11]. They found that IUGR children had lower quotient of intelligence and more frequent neuropsychological difficulties.

During life in the uterus, the malfunction of the placenta is the major cause of the under nutrition of the developing fetus [28]. The reduced supply of nutrients to the fetus from the placenta prevents it from getting his/her complete growth potential [9]. Barker et al. (1989) proposed that the development of cardiovascular disease is initiated by unfavourable conditions during intrauterine fetal life and adverse environment during early childhood [3]. This hypothesis has been reinforced by epidemiological evidences of association between birth weight and risk of cardiovascular disease $[15,21]$. The correlation between cardiovascular disease and low birth weight has been replicated among both males and females in Europe, North America and India [4, 7]. Banci et al. (2009) described negative correlation between birth weight and risk of coronary artery disease in non-diabetic females [2]. Andersen and co-workers described that higher BMI in children with very low birth weight is associated with higher cardiac risk [1].

The mechanism of cardiovascular system regulation is mainly dependent on autonomic control of the nervous system. The altered autonomic cardiovascular control, such as increased pulse, hypertension and heart rate variability have been described in low birth adults $[19,29]$. Heart rate variability analysis is a well established non-invasive measure of synergic activity of the autonomic nervous system, which regulates the heartbeat dynamics [13]. Heart rate variability has been widely used to measure cardiac autonomic control both in physiologic and pathologic conditions during the last two decades [23]. In 1996, the Task Force of European Society of Cardiology (ESC) and North American Society of Pacing Electrophysiology (NASPE) published standards for HRV analysis proposing several time and frequency domain parameters for assessment of HRV [23].

The purpose of this study was to investigate systematically the autonomic balance in 9-10 years old normal and IUGR children using linear techniques (traditional time and frequency domain HRV measures) and also methods derived from nonlinear dynamics. We 
have examined the effect of birth weight and gender on HRV of normal and IUGR children. Most of the HRV measures for IUGR-1 children (birth weight $<2.5 \mathrm{~kg}$ ) were significantly different from both normal and IUGR-2 children (birth weight $\geq 2.5 \mathrm{~kg}$ ). We also noticed that girls had lower HRV than boys.

\section{Material and Methods}

\section{Data Sets}

This work is part of a study that started at Leicester Royal Infirmary (LRI) for investigating the long term effects of intrauterine growth retardation on the autonomic nervous system. In the original study, infants who were identified as IUGR either by serial ultrasound scans, which identified infants who had fetal abdominal girth two or more standard deviation below the mean, or who, by birth weight, were on or below the $2^{\text {nd }}$ centile for gestational age [17]. Only the recordings fulfilling inclusion criteria (Caucasian, Gestation age $\geq 37$ weeks, no Congenital and chromosomal problem, no epilepsy or diabetes in mother, evidence of IUGR) were used for subsequent analysis. After a complete medical examination, the 24 hour ECG of IUGR and normal children were recorded with a Lifecard CF ambulatory ECG recorder (Delmar-Reynolds Medical Limited, Hertford, UK). The subjects were advised to perform normal daily routines during recording and to keep a diary of all activities including sleep and wake timings. The ECG recording of all subjects were extracted by Pathfinder 700 series analysis system and examined for artefacts. Characteristics of the study population are described in table 1. The normal group consisted of 33 subjects, 21 male and 12 female, current age $8.96 \pm 0.72$ years (mean \pm standard deviation), birth weight $3.53 \pm 0.48$ $\mathrm{kg}$, current weight $32.87 \pm 6.13 \mathrm{~kg}$, and gestational age $39.15 \pm 0.91$ weeks. The IUGR groups consisted of 37 subjects (16 male and 21 female), current age (9.31 \pm 0.62 years), birth weight $(2.58 \pm 0.42 \mathrm{~kg})$, current weight $(28.82 \pm 5.90 \mathrm{~kg})$ and gestational age $(38.95 \pm 1.37$ weeks $)$. There was no significant difference between the gestational ages of normal and IUGR groups, so we divided IUGR children into two groups on the basis of their birth weights. The low birth weight group IUGR-1 comprising of children having birth weight less than $2.5 \mathrm{~kg}$ and IUGR-2 having birth weight greater than $2.50 \mathrm{~kg}$. It is clear from table 1 that, by any criterion, IUGR children do not catch up with normal children in height or weight in the first decade of their life. IUGR-1 children gain weight at a faster rate (weight gain centile $37.37 \pm 25.21)$ than both IUGR-2 $(32.09 \pm 28.48)$ and normal children $(16.11 \pm 33.69)$. The 
children in the normal group have higher tendency to towards obesity, with $50 \%$ of them above the $85^{\text {th }}$ percentile for BMI. In our study there were more illness in IUGR-1 children (50\%) as compared to IUGR-2 (29\%) and normal children (12\%). The commonest medical condition was mild asthma, but there were two cases with autism, one child with epilepsy and two children with moderately delayed development.

\section{HRV Parameters}

There are many ways of assessing HRV and a comprehensive list of measures investigated since 1960 are explained in detail by the Task Force for European Society of Cardiology the North American Society of Pacing Electrophysiology [23]. In this study, both linear (time and frequency domains) and nonlinear HRV parameters were analysed.

\section{Linear HRV Techniques}

Linear time domain parameters included those measures derived from direct measurement of RR intervals (SDNN and SDANN) and from RR-intervals differences (RMSSD, NN50 and pNN50). Standard deviation of all normal to normal RR intervals (SDNN) is the most commonly used index for HRV analysis. SDNN can be calculated for short periods between $30 \mathrm{~s}$ and 5 min duration (short term variability) or for long periods (24 hours) as a measure of long term variation [23]. In practice it is inappropriate to compare SDNN measures of recordings of different duration and standardized durations of recordings have been suggested [23]. Other commonly used statistical index SDANN (standard deviation of average $\mathrm{NN}$ intervals calculated over 5-min intervals within the entire period of recording) is a measure of long term variation. The most commonly used measures of short term variation derived from interval differences include RMSSD (square root of mean squared differences of consecutive NN intervals), NN50 (number of pairs of NN intervals differing by more than $50 \mathrm{~ms}$ ) and pNN50 (proportion derived by dividing NN50 by total number of NN intervals).

The linear frequency domain measures used for HRV analysis included low frequency (LF, 0.04-0.15 Hz), high frequency $(\mathrm{HF}, 0.15-0.4 \mathrm{~Hz})$ and the ratio of LF to HF power (LF/HR). The power spectral density was calculated using fast Fourier transform-based Welch periodogram method. Prior to the spectrum estimation the interbeat interval time series was converted into equidistantly sampled time series using cubic spline interpolation and resampling. The resampling frequency was $4 \mathrm{~Hz}$, which works well for human data. The sampled data was divided into overlapping segments, and the Fourier transform was 
calculated for each segment. The power spectrum was obtained by averaging the spectra of these segments. The number samples in each segment was 256 with $50 \%$ overlapping. This decreases the variance of the spectral estimate.

\section{Nonlinear HRV Techniques}

The nonlinear behaviour of HRV was investigated using Poincaré plots, Approximate Entropy and Sample Entropy [20, 26, 27]. A Poincaré plot is a quantitative visual tool that represents correlation between successive RR intervals [20]. A common approach to quantitatively summarize the shape is to fit an ellipse to the plot $[14,22]$. The ellipse is oriented according to the line of identity. A standard Poincaré plot and its two basic two basic descriptors SD1 and SD2 are shown in the figure 1. SD1 measures dispersion of points perpendicular to the line of identity, describes short term variability. SD2 measures dispersion of points along the line of identity, describes long term variability.

Approximated Entropy (ApEn) analysis is a measure of irregularity or randomness of the signal that quantifies the predictability of the fluctuations in the signal [26]. Large values of ApEn indicate high complexity and smaller values of ApEn indicate a more regular signal. The values of ApEn depend on two factors, the length of the vectors (m), and the tolerance $(r)$, that is, ApEn can be written as $\operatorname{ApEn}(m, r)$. The tolerance $r$ is the percentage of standard deviation (SD) of the original time series (for HRV 10\%-25\% of standard deviation). Two patterns are similar if the difference between any pair of corresponding measurements in the pattern is less than $r$. The sample entropy (SamEn) is a modification of the ApEn algorithm in which self matches are excluded [27]. The SamEn is less dependent on the time series length and provides reliable and reproducible results. In this study the parameters used for the computation and ApEn and SamEn were $m=2$ and $r=0.2$. These values of $m$ and $r$ were chosen based on the results of previous studies by Pincus indicating good statistical validity for approximate entropy [26].

\section{Results}

The linear and nonlinear time series analysis techniques have been applied to interbeat interval time series of 33 normal and 37 IUGR 9 to 10 years old children. Wilcoxon rank sum test was used to find the significant difference between the groups with Bonferroni correction for repeated tests. In table 2, the results of HRV measures for normal and IUGR subjects are shown. The HRV decreased for IUGR subjects, but no significant difference was found 
between normal and IUGR subjects. The influences of birth weight and gender on HRV measures were also examined.

The results of linear and nonlinear HRV measures after dividing the IUGR cases according to birth weight are summarized in table 3. The time domain parameter of overall HRV, SDNN showed a significant difference between normal $V$ s. IUGR-1 $(\mathrm{p}=0.0129)$ and IUGR-1 Vs. IUGR-2 children ( $\mathrm{p}=0.0019)$, however, SDNN was not statistically significant in Normal Vs. IUGR-2 ( $\mathrm{p}=0.138)$. SDANN was statistically smaller in IUGR-1 children than both normal and IUGR-2children $(\mathrm{p}=0.0322$ and $0.0008 \mathrm{p}=$ respectively). Similar results were obtained for RMSSD for IUGR-1 Vs. normal and IUGR-1 and IUGR-2 ( $\mathrm{p}=0.0010$ and $\mathrm{p}=0.0016$ respectively). NN50 and pNN50 were significantly different between IUGR-1 $V s$. IUGR-2, but revealed no significant difference between normal $V s$. IUGR-1 and normal $V s$. IUGR-2. The frequency domain HRV measures LF, HF and LF/HF were significantly smaller in IUGR-I than both normal $(\mathrm{p}=0.0217, \mathrm{p}=0.0321$ and $\mathrm{p}=0.0384$ respectively) but LF and HF were significantly smaller between IUGR-1 Vs. IUGR ( $\mathrm{p}=0.0012$ and 0.0046). None of the linear HRV measures showed significant difference between normal and IUGR-2 children.

The nonlinear measures used for quantifying the dynamics of heart rate signals of normal and IUGR groups include Poincaré plots, approximate entropy and sample entropy. Two Poincaré plot indices SD1 (dispersion of points perpendicular to the line of identity) and SD2 (dispersion of points along the line of identity) were computed. The value of SD1 was significantly smaller between IUGR-1 $V s$. IUGR-2 $(\mathrm{p}=0.0011)$ but not between normal $V s$. IUGR-1 $(\mathrm{p}=0.1442)$. SD2 revealed significant difference between normal Vs. IUGR-1 $(\mathrm{p}=0.0209)$ and within IUGR groups $(\mathrm{p}=0.0033)$ but not for normal Vs. IUGR-2.

The nonlinear complexity measures, approximate entropy and sample entropy were computed by setting length of vector $\mathrm{m}=2$, tolerance $\mathrm{r}=20 \%$ of standard deviation of the time series. ApEn was not statistically significant between any of the groups ( $\mathrm{p}=0.4423$ normal $V s$. IUGR-1, $\mathrm{p}=0.1198$ IUGR-1 $V s$. IUGR-2 and $\mathrm{p}=0.5003$ normal $V s$. IUGR-2). Sample entropy showed significant difference normal Vs. IUGR-1 and within IUGR groups $(\mathrm{p}=0.0209$ and 0.0328 respectively), but not for normal Vs. IUGR-2.

The HRV parameters for studying gender related differences in normal and IUGR children are presented in table 4. A decrease in heart rate variability was found in both normal and IUGR female children. SDNN, LF and SD2 were significantly smaller in female subjects $(\mathrm{p}=0.0360, \mathrm{p}=0.04$ and $\mathrm{p}=0.0369$ respectively), however no other HRV parameter reached statistically significant level in normal children based on gender. 


\section{Discussion}

During intrauterine life, malfunctioning of fetal supply, which includes placenta, is the major cause of fetus under nutrition [28]. The fetus survives by metabolic and cardiovascular adaptations. The control mechanism of cardiovascular system depends on the autonomic nervous system involving mediation of both sympathetic and parasympathetic branches. In growth restricted children, altered cardiovascular control such as increased pulse rate [25] altered blood pressure and heart rate fluctuations [19, 29] have been observed. Numerous animal studies support the connection of unfavourable prenatal environment and alterations in the sympathetic autonomic balance [18, 24]. Flanagan and co-workers found inverse correlation between adult resting pulse rate (sympathetic activity index) and birth weight [10]. In IUGR adolescents, an increased cardiac sympathetic nerve activity was observed [16].

The adverse environment during fetal life may alter the development of the autonomic nervous system, which appears to persist in postnatal life. Heart rate variability (HRV) analysis is a valuable non-invasive measure of autonomic control, which regulates heart rate dynamics. During last three decades a large number of studies both in physiological and pathological condition show the power of HRV as quantitative marker of the activity of the cardiovascular control system [23]. This study was carried out to find the association between intrauterine growth retardation and cardiovascular disease, as well as the early identification and possible prevention of illness in later life. The cardiac autonomic activity was assessed using linear and nonlinear heart rate variability from 24 hour ECG recordings of IUGR and normal 9 to 10 years old children. We found no significant alteration in HRV measures of normal and IUGR (combined IUGR-1 and IUGR-2) children.

The birth weight of only one normal child was less than $2.5 \mathrm{~kg}$ and more than $50 \%$ IUGR subjects have birth weight less than $2.5 \mathrm{~kg}$. In order to examine the effect of birth weight on HRV parameters, we divided IUGR subjects into IUGR-1 and IUGR-2. SDNN, SDANN, RMSSD, LF, HF, SD2 and SamEn showed significant differences between IUGR-1 $V s$. Normal as well as IUGR-1 and IUGR-2. NN50, pNN50 and SD1 ratio were statistically significant within IUGR groups but not in normal Vs. IUGR-1 children. None of the HRV measures except SD1 revealed significant difference between IUGR-2 and normal children. Table 3 shows that HRV parameters are similar for normal and IUGR-2 subjects and are significantly different for IUGR-1. Our results demonstrate significantly altered autonomic control of low birth weight IUGR children (IUGR-1). The altered autonomic control of low 
birth weight IUGR children is in accordance with Baker's hypothesis of fetal origin of adult disease [3]. The epidemiological evidence of this hypothesis has been provided in various studies $[6,15,21]$. In different studies among male and female in Europe, North America and India, the association of low birth weight and cardiovascular disease has been described [4].

The effect of gender on HRV indices was studied and we noticed a decrease in HRV in both normal and IUGR female children. In normal children, SDNN, LF and SD1 showed significant sex difference but no HRV measure reached to significant level on gender basis in IUGR children. The decrease in HRV parameters for female children is consistent with the study of [5], who found that the female population presents lower overall HRV compared with the male population.

Our finds indicate that disturbances in ANS function reflected by reduced heart rate variability may represent one of the pathways to negative outcome of the cardiovascular system and hence the risk of coronary heart disease in low birth weight IUGR children. Andersen et al. (2010), found that birth weight and BMI at the age of seven appears to exercise mutually independent effects on the risk of cardiovascular disease [1]. They described that children with a combination of low birth weight and relatively high BMI had a cardiac risk of $44 \%$. The present study showed an inverse relation of birth weight and negative outcome of the cardiovascular system in growth retarded children, however, as the BMI of low birth weight IUGR children is small, we did not study the relation of BMI and risk of coronary heart disease.

The established cardiovascular risk in low birth weight IUGR children, concerns the underlying physiological mechanisms. The hypothesis that this is driven by a stress/autonomic response has been made before. The differences between normal and low birth weight IUGR Children provide further evidence for this at an age in which clinical manifestation of disease are not yet apparent. Our findings implicate further follow-up study as the child progresses to second decade of his life.

\section{Conclusions}

The association of fetal growth retardation and development of cardiovascular disease has been extensively replicated. The cardiovascular system regulation strongly depends on the autonomic control of the nervous system. Heart rate variability is a valuable non-invasive marker of cardiac autonomic balance. In this study, linear and non-linear HRV parameters were computed for seventy 9 to 10 year old normal and IUGR children. The direct comparison of HRV parameters between normal and IUGR children showed no significant 
difference between the two groups. We also examined the effect of birth weight and gender on HRV parameters. The findings revealed that most of HRV measures showed significant difference between Normal and low birth weight IUGR children and also within IUGR groups. The decrease in HRV depicts the altered autonomic control in low birth weight IUGR children as compared with normal and IUGR-2 children. Three HRV measures (SDNN, LF and SD1) showed significant gender specific differences in the normal cohort. It is suggested that low birth weight in IUGR children can have adverse implications for the autonomic nervous system.

\section{Acknowledgements}

We are grateful to Higher Education Commission (HEC) of Pakistan for financial support.

\section{References}

1. Andersen LG, Ängquist L, Eriksson JG, Forsen T, Gamborg M, Osmond C, Baker JL and Sorensen TIA (2010) Birth weight, childhood body mass index and risk of coronary heart disease in adults: Combined historical cohort studies. Plos One 5(11): e14126

2. Banci M, Saccucci P, Dofcaci A, Sansoni I, Magrini A, Bottini E and Gloria-Bottini F (2009) Birth weight and coronary artery disease. The effect of gender and diabetes $5(3): 244-248$

3. Barker DJP, Osmond C, Golding J, Kuh D and Wadsworth MEJ (1989) Growth in utero, blood pressure in childhood and adult life, and mortality from cardiovascular disease. Br Med J 298: 564-567

4. Barker DJP (2004) The developmental origin of chronic adult disease. Acta Paediatr Suppl 446: 26-33

5. Beckers F, Ramaekers D and Aubert AE (2001) Gender related differences in nonlinear indices of heart rate variability Memories II Latin American Congress of Engineering and Biomedical Havana. 950:7132-57-5

6. Eriksson JG, Forsen T, Tuomilehto J, Osmond C and Barker DJP (2000) Fetal and childhood growth and hypertension in adult life. J Clin Endocrinol Metab 84: 790794

7. Eriksson JG, Forsen T, Tuomilehto J, Osmond C and Barker DJP (2001) Early growth and coronary heart disease in later life; longitudinal study. Br Med J 322: 949-953 
8. Fairley L and Leyland AH (2006) Social class inequalities in perinatal outcomes: Scotland 1980-2000. J Epidmiol Community Health 60: 31-36

9. Ferrario M, Signorini MG and Magenes G (2009) Complexity analysis of fetal heart rate variability: early identification of severe intrauterine growth-restricted fetuses. Med Biol Eng Comput 47: 911-919

10. Flanagan DE, Vaile JC, Petley GW, Moore VM, Godsland IF, Cockington RA, Robinson JS and Phillips DI (1999). The autonomic control of heart rate and insulin resistance in young adults. J Clin Endocrinol Metab 84: 1263-1267

11. Geva R, Eshel R, Leitner Y, Valevski AF and Harel S (2006) Neuropsychological outcome of children with intrauterine growth restriction: A 9-year study. Pediatrics 118:91-100

12. Goldenberg RL and Culhane JF (2007) Low birth weight in the United States. Am J Clin Nutr 85:584S-590S

13. Guzzetti S, Dassi S, Pecis M, Casati R, Masu AM, Longoni P, Tinelli M, Cerutti S, Pagani M and Malliani A (1991) Altered pattern of circadian neural control of heart period in mild hypertension. J Hypertens 9: 831-838

14. Huikuri H V, Seppanen T, Koistinen M, Juhani A, Ikaheimo M J, Castellanos A and Myerburg R J (1996) Abnormalities in beat-to-beat dynamics of heart rate before spontaneous onset of life-threatening ventricular tachyarrhythmias in patients with prior myocardial infarction. Circulation 93: 1836-1844

15. Huxley RR, Shiell AW and Law CM (2001). The role of size of birth and postnatal catch-up growth in determining systolic pressure: a systematic review of the literature. J Hypertension 18: 815-831

16. Ijzerman RG, Stehouwer CD, de Geus EJ, van Weissenbruch MM, Delemarre-van de Waal HA and Boomsma DI (2003) Low birth weight is associated with increased sympathetic activity: dependence on genetic factors. Circulation 108: 566-571

17. Jackson JA Wailoo MP, Thompson JR and Petersen SA (2004) Early physiological development of infants with intrauterine growth retardation. Arch Dis Child Fetal Neonatal 89:F46-F50

18. Jansson T and Lambert GW (1999) Effect of intrauterine growth restriction on blood pressure, glucose tolerance and sympathetic nervous system activity in the rat at 3-4 months of age. J Hypertens 17: 1239-1248 
19. Jones A, Beda A, Ward AM, Osmond C, Phillips DI, Moore VM and Simpson DM (2007) Size at birth and autonomic function during psychological stress. Hypertension 49: $548-555$

20. Kamen P W, H H K and Tonkin a M (1996). Poincare plot of heart rate variability allows quantitative display of parasympathetic nervous activity in humans. Clini Sci 91(2): 201-208

21. Law CM and Shiell AW (1996) Is blood pressure inversely related to birth weight? The strength of evidence from a systematic review of the literature. J Hypertens 14 : 935-941

22. Marciano F, Migaux M L, Acanfora D, Furgi G and Rengo F (1994) Quantification of Poincare maps for the evaluation of heart rate variability. Comput in Cardio 21: 577580

23. Malik M et al (1996) Task force of the European society of cardiology and north American society of pacing electrophysiology: Heart rate variability: standards of measurement, physiological interpretation and clinical use. Circulation 93: 1043-1065

24. Oyama K, Padbury J, Chappell B, Martinez A, Stein H and Humme J (1992) Single umbilical artery ligation-induced fetal growth retardation: effect on postnatal adaptation. Am J Physiol Endocrinol Metab 263: E575-E583, 1992

25. Phillips DI and Barker DJ (1997). Association between low birth weight and high resting pulse in adult life: is the sympathetic nervous system involved in programming the insulin resistance syndrome? Diabet Med 14: 673-677

26. Pincus S M (1991) Approximate entropy as a measure of system complexity. In Proceedings of the National Academy of Sciences, pp. 2297-2301. USA

27. Richman J S and Moorman J R (2000). Physiological time-series analysis using approximate entropy and sample entropy. Am J Physiol Heart and Circ Physiol 278: H2039-H2049

28. Schaäffer L, Burkhardt T, Müller-Vizentini D, Rauh M, Tomaske M, Meith RA, Bauersfeld U and Beinder E (2008) Cardiac autonomic balance in small-forgestational-age neonates. Am J Physiol Heart Circ Physiol 294: H884-H-890

29. Ward AM, Moore VM, Steptoe A, Cockington RA, Robinson JS and Phillips DI (2004). Size at birth and cardiovascular responses to psychological stressors: evidence for prenatal programming in women. J Hypertens 22: 2295-2301

30. World Health Organization (1993) International statistics classification of diseases and related health problems. $10^{\text {th }}$ revision, Vol. 2. Geneva, Switzerland: WHO 
Table 1

\begin{tabular}{|l|c|c|c|}
\hline & Normal & IUGR-1 & IUGR-2 \\
\hline Recordings & 33 & 20 & 17 \\
Birth Weight $(\mathrm{kg})$ & $3.53 \pm 0.48$ & $2.29 \pm 0.19$ & $2.92 \pm 0.36$ \\
Gender (Male/Female) & $21 / 12$ & $8 / 12$ & $8 / 9$ \\
Current Weight $(\mathrm{kg})$ & $32.87 \pm 6.13$ & $28.13 \pm 4.74$ & $29.64 \pm 6.89$ \\
Weight Gain $(\mathrm{kg})$ & $29.34 \pm 6.13$ & $25.83 \pm 4.75$ & $26.72 \pm 6.88$ \\
Weight Gain Centile & $16.11 \pm 33.69$ & $37.37 \pm 25.21$ & $32.09 \pm 28.48$ \\
Current Height (cm) & $133.86 \pm 5.00$ & $131.00 \pm 5.84$ & $132.18 \pm 7.01$ \\
Current Height Centile & $57.91 \pm 25.75$ & $34.17 \pm 25.04$ & $33.25 \pm 25.18$ \\
Current BMI (kg/m $\left.{ }^{2}\right)$ & $81.21 \pm 2.52$ & $16.38 \pm 2.55$ & $16.77 \pm 2.58$ \\
Current BMI Centile & $72.72 \pm 28.40$ & $42.75 \pm 29.29$ & $50.25 \pm 29.30$ \\
\hline
\end{tabular}


Table 2

\begin{tabular}{|l|l|l|r|}
\hline \multicolumn{1}{|c|}{$\begin{array}{c}\text { HRV } \\
\text { Measures }\end{array}$} & \multicolumn{1}{|c|}{ Normal } & \multicolumn{1}{c|}{ IUGR } & p-value \\
\hline SDNN (ms) & $156.82 \pm 28.24$ & $152.82 \pm 35.21$ & 0.6053 \\
SDANN (ms) & $77.65 \pm 20.46$ & $76.87 \pm 21.33$ & 0.8766 \\
RMSSD (ms) & $70.32 \pm 28.86$ & $69.58 \pm 33.62$ & 0.9226 \\
NN50 (ms) & $28290 \pm 11179$ & $29870 \pm 12830$ & 0.5867 \\
pNN50 (\%) & $23.55 \pm 10.51$ & $24.64 \pm 11.99$ & 0.6893 \\
LF (ms $\left.{ }^{2}\right)$ & $1966 \pm 969$ & $1840 \pm 907$ & 0.5742 \\
HF (ms $\left.{ }^{2}\right)$ & $2704 \pm 2177$ & $2678 \pm 2724$ & 0.9647 \\
LF/HF & $0.92 \pm 0.38$ & $1.16 \pm 0.78$ & 0.1542 \\
SD1 (ms) & $49.72 \pm 20.41$ & $54.81 \pm 28.28$ & 0.3962 \\
SD2 (ms) & $215.63 \pm 37.42$ & $205.16 \pm 53.32$ & 0.3507 \\
ApEn & $1.14 \pm 0.08$ & $1.16 \pm 0.09$ & 0.1908 \\
SamEn & $1.07 \pm 0.08$ & $1.10 \pm 0.13$ & 0.1269 \\
\hline
\end{tabular}


Table 3

\begin{tabular}{|c|c|c|c|c|c|c|}
\hline \multirow[b]{2}{*}{$\begin{array}{c}\text { HRV } \\
\text { Measures }\end{array}$} & \multirow[b]{2}{*}{ Normal } & \multirow[b]{2}{*}{ IUGR-1 } & \multirow[b]{2}{*}{ IUGR-2 } & \multicolumn{3}{|c|}{ p-value } \\
\hline & & & & $\begin{array}{c}\text { Normal } \\
\text { Vs. } \\
\text { IUGR-1 } \\
\end{array}$ & $\begin{array}{c}\text { IUGR-1 } \\
\text { Vs. } \\
\text { IUGR-2 } \\
\end{array}$ & $\begin{array}{c}\text { Normal } \\
V s . \\
\text { IUGR-2 } \\
\end{array}$ \\
\hline $\mathrm{SDNN}(\mathrm{ms})$ & $156.81 \pm 28.24$ & $136.99 \pm 25.22$ & $171.45 \pm 36.78$ & 0.0129 & 0.0019 & 0.138 \\
\hline SDANN (ms) & $77.65 \pm 20.46$ & $66.66 \pm 11.27$ & $88.87 \pm 24.28$ & 0.0322 & 0.0008 & 0.091 \\
\hline RMSSD (ms) & $70.32 \pm 28.86$ & $53.72 \pm 18.78$ & $86.25 \pm 37.93$ & 0.0262 & 0.001 & 0.0681 \\
\hline $\mathrm{NN} 50(\mathrm{~ms})$ & $28290 \pm 11179$ & $25139 \pm 10115$ & $35435 \pm 13712$ & 0.308 & 0.0128 & 0.0534 \\
\hline pNN50 (\%) & $23.55 \pm 10.51$ & $19.83 \pm 8.87$ & $30.30 \pm 12.93$ & 0.1916 & 0.0063 & 0.0525 \\
\hline $\mathrm{LF}\left(\mathrm{ms}^{2}\right)$ & $1966 \pm 2214$ & $1418 \pm 456$ & $2336 \pm 1057$ & 0.0217 & 0.0012 & 0.2207 \\
\hline $\mathrm{HF}\left(\mathrm{ms}^{2}\right)$ & $2704 \pm 2178$ & $1548 \pm 1102$ & $4007 \pm 3433$ & 0.0321 & 0.0046 & 0.1078 \\
\hline $\mathrm{LF} / \mathrm{HF}$ & $0.92 \pm 0.38$ & $1.23 \pm 0.66$ & $1.03 \pm 0.88$ & 0.0384 & 0.4379 & 0.5602 \\
\hline $\mathrm{SD} 1$ (ms) & $49.72 \pm 20.41$ & $41.56 \pm 17.64$ & $70.41 \pm 30.85$ & 0.1440 & 0.0011 & 0.0066 \\
\hline $\mathrm{SD} 2(\mathrm{~ms})$ & $215.63 \pm 37.42$ & $182.37 \pm 39.32$ & $231.97 \pm 56.11$ & 0.0209 & 0.0033 & 0.2249 \\
\hline ApEn & $1.14 \pm 0.08$ & $1.17 \pm 0.09$ & $1.15 \pm 0.08$ & 0.4423 & 0.1198 & 0.5003 \\
\hline SamEn & $1.07 \pm 0.08$ & $1.13 \pm 0.13$ & $1.07 \pm 0.12$ & 0.0209 & 0.0328 & 0.8684 \\
\hline
\end{tabular}


Table 4

\begin{tabular}{|l|l|l|l|l|l|l|}
\hline \multirow{2}{*}{$\begin{array}{c}\text { HRV } \\
\text { Measures }\end{array}$} & \multicolumn{1}{|c|}{ Male } & \multicolumn{1}{|c|}{ Female } & p-value & \multicolumn{1}{c|}{ Male } & \multicolumn{1}{c|}{ Female } & p-value \\
\cline { 2 - 7 } SDNN (ms) & $164.52 \pm 30.14$ & $143.34 \pm 18.87$ & 0.0360 & $158.84 \pm 36.96$ & $148.24 \pm 32.98$ & 0.3721 \\
SDANN (ms) & $82.39 \pm 23.13$ & $69.35 \pm 11.27$ & 0.0778 & $79.92 \pm 23.40$ & $74.54 \pm 19.18$ & 0.4557 \\
RMSSD (ms) & $76.12 \pm 32.32$ & $60.18 \pm 18.64$ & 0.1289 & $69.95 \pm 32.69$ & $69.30 \pm 34.34$ & 0.9544 \\
NN50 (ms) & $29502 \pm 12899$ & $26169 \pm 7303$ & 0.4187 & $30555 \pm 12723$ & $29348 \pm 12890$ & 0.7813 \\
pNN50 (\%) & $24.97 \pm 11.51$ & $21.07 \pm 8.37$ & 0.3136 & $25.90 \pm 12.48$ & $23.68 \pm 11.49$ & 0.5848 \\
LF (ms $\left.{ }^{2}\right)$ & $2225 \pm 1095$ & $1513 \pm 449$ & 0.0400 & $2079 \pm 1081$ & $1658 \pm 682$ & 0.1642 \\
HF (ms $\left.{ }^{2}\right)$ & $3086 \pm 2474$ & $2036 \pm 1380$ & 0.1873 & $2753 \pm 2677$ & $2620 \pm 2760$ & 0.8855 \\
LF/HF & $0.88 \pm 0.35$ & $0.99 \pm 0.44$ & 0.4444 & $1.11 \pm 0.58$ & $1.16 \pm 0.89$ & 0.8604 \\
SD1 (ms) & $53.82 \pm 22.86$ & $42.55 \pm 13.18$ & 0.1289 & $54.65 \pm 27.16$ & $54.94 \pm 29.14$ & 0.9760 \\
SD2 (ms) & $225.79 \pm 39.52$ & $197.85 \pm 26.33$ & 0.0369 & $213.49 \pm 54.89$ & $198.82 \pm 51.09$ & 0.4148 \\
ApEn & $1.13 \pm 0.08$ & $1.14 \pm 0.07$ & 0.7209 & $1.18 \pm 0.08$ & $1.15 \pm 0.09$ & 0.3318 \\
SamEn & $1.05 \pm 0.09$ & $1.09 \pm 0.08$ & 0.2497 & $1.13 \pm 0.12$ & $1.08 \pm 0.13$ & 0.2339 \\
\hline
\end{tabular}

